Revista de Educação e Pesquisa em Contabilidade

Journal of Education and Research in Accounting

Revista de Educación e Investigatión en Contabilidad
REPeC, Brasília, v. 6, n. 3, art. 5, p. 299-315, jul./set. 2012

Disponível online em www.repec.org.br

ISSN 1981-8610

\title{
Generalização algébrica da DFC: reflexões por meio de um algoritmo algébrico
}

\author{
Antonio Geloneze Neto \\ Bacharel em Ciências Contábeis (FEA-USP) \\ Professor Aposentado da Universidade Estadual Paulista. \\ Endereço: Rua Armando D'Almeida, 170 - Jardim Rizzo - São Paulo/SP - Cep 05587-010 \\ E-mail: ageloneze@gmail.com
}

\section{José Roberto Kassai}

Doutor em Controladoria e Contabilidade (FEA-USP)

Professor Titular da Universidade de São Paulo.

Endereço: Av. Prof.Luciano Gualberto, 908 - Cidade Universitária - São Paulo/SP - Cep 05508-900

E-mail:jrkassai@usp.br

\section{Resumo}

A Demonstração de Fluxos de Caixa (DFC) passou a ser um relatório obrigatório pela contabilidade a partir de $1^{\circ}$ de janeiro de 2008 para todas as empresas de capital aberto ou com patrimônio líquido superior a dois milhões de reais e, dessa forma, torna-se mais um importante relatório para a tomada de decisões gerenciais. Este trabalho tem por objetivo propor uma generalização algébrica para a DFC. Papéis de trabalho podem contribuir para fechar uma lacuna didática no ensino da DFC e produzir o método indireto e o método direto, lado a lado com sua equivalência destacada, em uma mesma matriz por meio de algoritmos algébricos. A pesquisa é de natureza normativa e enfatiza a transversalidade entre a Contabilidade e a Matemática, mostrando que os relatórios contábeis e suas estruturas podem ser vistos como matrizes e sujeitas a deduções algébricas sobre os eventos registrados por meio das partidas dobradas. Como resultado, pôde-se demonstrar um algoritmo matemático com matrizes e submatrizes e um roteiro no formato de papéis de trabalho, compatíveis com as orientações normativas para a DFC na legislação brasileira, que permite uma DFC clara, segura e efetiva.

Palavras-chaves: Algoritmo algébrico - DFC - Partidas dobradas

Editado em Português, Inglês e Espanhol. Versão original em Português.

Recebido em 09/05/11. Pedido de Revisão em 19/9/11 e 13/10/11. Resubmetido em 23/11/11. Aceito em 27/01/2012 por Valcemiro Nossa (Editor). Publicado em 14/09/12. Organização responsável pelo periódico: CFC/FBC/ABRACICON.

Copyright (C) 2012 REPEC. Todos os direitos, até mesmo de tradução, são reservados. É permitido citar parte de artigos sem autorização prévia, desde que seja identificada a fonte. 


\section{INTRODUÇÃO}

A ideia de debitar $x$ na conta A e creditar $x$ na conta B, toda vez que $x$ for o valor de um lançamento no Livro Diário, é um notável modelo algébrico, criado por contadores desconhecidos, formalizado principalmente por Luca Pacioli (PACIOLI, 1494), que escreveu na parte do Tratactus de Computis et Scripturis ou Contabilidade por Partidas Dobradas: “... à teoria contábil do débito e do crédito corresponde a teoria dos números positivos e negativos."

Isto leva logicamente à equação fundamental da contabilidade:

$$
\mathbf{A}=\mathbf{P}+\mathbf{P L}
$$

Está bem estabelecida a aplicabilidade da álgebra na própria fundação da contabilidade, assim como sua influência na elaboração de demonstrativos contábeis formalizados em matrizes de modelos algébricos. Os usuais razonetes e demonstrativos contábeis são impensáveis sem sua estrutura algébrica matricial. Jean Le Rond D'Alembert (1717 - 1783), já chamava a atenção para o potencial de aplicação da álgebra (MACHALE, 1993), reconhecendo que: "Algebra is generous: she often gives more than is asked for."

(a álgebra é generosa: ela frequentemente dá mais do que se lhe pede). Não poderia ser diferente com a Contabilidade, principalmente, a partir da álgebra das partidas dobradas.

Há uma lacuna na didática do ensino da DFC. Falta um método claro, seguro e efetivo para a elaboração desta demonstração. Examine-se, por exemplo, Marques; Carneiro \& Kuhl, 2008, Campos, 1999 e Fipecafi, 2010. Basicamente, esta literatura oferece exemplos de DFC. O aprendiz de Contabilidade terá, certamente, dificuldades em elaborar a DFC seguinte àquela do exemplar fornecido. Um dos principais problemas é o "mistério" que envolve, aparentemente, a equivalência entre a conciliação do lucro líquido no método indireto e os pagamentos e recebimentos operacionais do método direto. $\mathrm{O}$ algoritmo proposto aqui tem o objetivo de ser um método claro, seguro e efetivo para o aprendiz de Contabilidade.

Este trabalho propõe um método algébrico, materializado em papéis de trabalho para a DFC, para os aprendizes visualizarem as duas formas da DFC como sendo apenas duas expressões equivalentes de um mesmo invariante. Consequentemente, não é natural imaginá-los como separados e independentes, como parece sugerido na literatura em geral. Não são abordadas aqui as imprecisões relacionadas à definição de atividades operacionais que estão, evidentemente, relacionadas às imprecisões da definição de atividades de investimento e de financiamento. Em Marques, Carneiro e Kuhl (2008), há uma descrição meticulosa do Pronunciamento CPC 03 tratando o problema de uma maneira global.

Em suma, o objetivo desta pesquisa é oferecer um método de elaboração de DFC didaticamente mais eficiente e eficaz, livre de exemplares particulares que sempre serão insuficientes para diferentes planos de contas, por meio de análise algébrica e um algoritmo.

\section{FUNDAMENTAÇÃO TEÓRICA}

A DFC passou a ser obrigatória para todas as sociedades de capital aberto ou com um patrimônio líquido superior a dois milhões de reais, por força da Lei n. ${ }^{\circ}$ 11.638/2007 com Deliberação CVM 547/2008 aprovando o CPC 03.

A Lei n. ${ }^{\circ} 11.638 / 2007$ (Lei n. ${ }^{\circ} 11.638$ ), artigo $1^{\circ}$, altera a antiga nova Lei das S/A e dá nova redação ao artigo 176 da Lei n. ${ }^{\circ}$ 6.404/1976 (Lei n. ${ }^{\circ}$ 6.404), incluindo o inciso IV com nova demonstração contábil obrigatória: a DFC.

A Deliberação n. 547 (CVM 547), de 13 de agosto de 2008, publicada no DOU de 15/8/208, aprovou o CPC 03 (CPC 03), que trata da DFC. 


\section{repec}

O CPC 03 - DFC correlaciona-se ao pronunciamento IAS 7 do International Accounting Standards Board (IASB) (IAS 7) e, em suas vinte e seis páginas, discorre sobre conteúdo, objetivos, alcance, benefícios das informações dos fluxos de caixa, definições, caixa e equivalentes de caixa, apresentação em atividades operacionais-investimento-financiamento, divulgações e outras instruções de como se elaborar a DFC de instituições financeiras e não financeiras e seus modelos direto e indireto. (http://www.cpc.org.br, fev/2010)

Como ainda é um relatório recente, em vigor desde 01/01/2008, a DFC tem sido elaborada nas empresas e escolas de acordo com as habilidades de cada um. O método direto, apesar de aparentemente mais fácil de se elaborar, na verdade requer precisão dos sistemas de contabilidade disponíveis e, por isso, muitas vezes tem sido elaborado por meio de planilhas eletrônicas e por tentativas e erros (KASSAI, 2009).

Assim, a abordagem da DFC proposta aqui enfatiza as propriedades algébricas de [2] e [3], em seu caráter invariante. A partir delas, podem-se utilizar matrizes-colunas com somas invariantes para se demonstrar os fluxos de caixa por meio de um algoritmo justificado algebricamente, que fornece, ao mesmo tempo, os métodos direto e indireto mencionados no CPC 03.

A própria Evolução é vista pela ciência da complexidade (BEINHOCKER, 2006, p. 317) como um algoritmo de aprendizagem: "Evolution is a knowledge-creation machine - a learning algorithm".

A literatura contábil, pelo menos aquela que se pretende didática, deve apresentar, com clareza e distinção, incorporando esta recomendação feita por Descartes para todas as ciências (DESCARTES, 1637), papéis de trabalho acompanhados de algoritmos de preenchimento que produzam, sistematicamente e seguramente, matrizes das quais se possa extrair naturalmente os demonstrativos contábeis. Em geral, os livros utilizam exemplos numéricos para explicar os demonstrativos, mas basta aparecer uma empresa que não tenha os mesmos grupos de contas para que uma dificuldade interrompa imediatamente a capacidade de se elaborar o relatório com a mesma eficiência e eficácia.

Uma característica desse modelo algébrico é a consideração de que os dois métodos para a elaboração da DFC (direto e indireto) estão conectados algebricamente, não apenas porque duas de suas três estruturas matriciais coincidem (investimento e financiamento), mas principalmente porque possuem simetrias algébricas intrínsecas decorrentes da álgebra das partidas dobradas.

Os lançamentos no Livro Diário são as "partículas fundamentais" da Contabilidade. A definição de Método Direto é clara e distinta: somente lançamentos dos tipos "pagamento" e "recebimento" devem ser evidenciados na DFC. Analogamente, uma definição do Método Indireto deve se pronunciar, clara e distintamente, sobre quais lançamentos precisam ser evidenciados. O problema se restringe à primeira das três estruturas matriciais da DFC, o conjunto das atividades operacionais, uma vez que para as atividades de investimento e de financiamento a definição é a mesma em ambos os métodos. É justamente a definição de conciliação do lucro líquido com o caixa que monopoliza a imprecisão; algebricamente são equivalentes eliminar a imprecisão da "conciliação do lucro líquido" e eliminar a imprecisão na escolha das contas cujas variações devem ser evidenciadas.

A dificuldade em elaborar a DFC não se esgota na dúvida por "onde começar?" e se estende em saber se "vai bater?", "por que não bateu?", "por que bateu" e "como posso saber se está certo?" e, finalmente, em saber como verificar facilmente e sistematicamente se a DFC está certa. Por isso, o algoritmo proposto possui a característica de ser algebricamente natural, o que possibilita a verificação de todas as relações envolvidas na DFC.

\section{METODOLOGIA}

Este artigo explora a álgebra diferencial de dois balanços consecutivos, expressa nas equações:

$$
\Delta \mathbf{A}=\Delta \mathbf{P}+\Delta \mathbf{P L}
$$




\section{repec}

$\Delta \mathbf{E q C x}=-\Sigma \Delta[$ contas do ativo $]+\Sigma \Delta[$ c. do passivo $]+\Sigma \Delta[$ c. do patrimônio líquido $]$

Onde:

$\Delta=$ diferença

$\mathrm{EqCx}=$ equivalente de caixa

$\Sigma=$ soma, combinada com uma estrutura matricial específica.

As equações [2] e [3] não são novidade para os contadores (MARQUES; CARNEIRO \& KUHL, 2008), embora talvez o seja a interpretação como invariantes algébricos derivados do invariante algébrico fundamental [1]. As equações [2] e [3] aparecem de um modo promissor, mas o potencial algébrico delas permanece intocado e o texto percorre uma trajetória de "exemplos" para expor a DFC. Exemplares são importantes no esclarecimento da DFC (MARQUES, CARNEIRO \& KUHL, 2008; CAMPOS, 1999; FIPECAFI, 2010), mas ela precisa também ser apresentada diretamente, sem subterfúgios, e se impor logicamente por si mesma.

No que concerne à abordagem científica, enfatizou-se a interdisciplinaridade, no sentido de que os relatórios contábeis são matrizes com certa estrutura, e a dedução algébrica, a partir da definição de partida dobrada, ambas tendo como guia do pensamento o método Cartesiano (DESCARTES, 1637) aplicável a todo sistema que se pretenda científico. Esta opção de abordagem científica ressalta a postura matemática de Luca Pacioli de fundamentar a contabilidade nas propriedades algébricas dos números positivos e negativos, subordina-se ao método filosófico de Descartes (DESCARTES, 1637) e acredita no potencial da álgebra segundo a visão de D'Alembert (MACHALE, 1993).

Quanto à metodologia, foi utilizada a pesquisa normativa, uma vez que ela sugere como deve ser a elaboração da DFC por meio de matrizes e na forma de papéis de trabalho.

Uma análise dos artigos e livros citados e relacionados ao tema da DFC comprova, por um lado, a aplicação das ideias desenvolvidas neste trabalho e, por outro lado, dá uma amostra da ausência das mesmas na literatura disponível.

Por exemplo, não é provável que o aprendiz aprenda a DFC pelo exemplar aritmético do importante Manual de Contabilidade Societária (FIPECAFI, 2010). Pode-se consultar todas as sete edições anteriores deste Manual para se verificar o mesmo fenômeno. Dificilmente a lógica de um exemplar se generaliza para qualquer outro plano de contas. Outros textos aqui citados estão afetados pela mesma dificuldade (MARQUES, CARNEIRO \& KUHL, 2008; CAMPOS, 1999).

A literatura estrangeira parece não fugir a esta regra (NURNBERG, 1989; DRTINA \& LARGAY, 1985). Há pesquisadores com vasta pesquisa envolvendo fluxos de caixa, mas o foco do assunto, além de não ser elementar como o do presente trabalho, é completamente diferente.

Por exemplo, na obra de Patrícia Dechow (DECHOW, 1994) encontramos diversos artigos abordando a questão interessante de mensuração do importante intangível "firm performance" para o qual ela utiliza os fluxos de caixa como indicadores mensuráveis. Em Dechow, Khotari, Watts (1998), os autores investigam o problema de prever fluxos de caixa por meio de um modelo matemático-estatístico envolvendo series temporais, um tema, portanto, longe de ser trivial e bem distante do tema elementar apresentado aqui apenas de entendimento claro e elaboração segura e efetiva da demonstração dos fluxos de caixa.

É provável ser bem difícil encontrar na literatura disponível, no país ou no exterior, artigos ou livros abordando a elaboração da DFC de um modo próximo ao adotado neste trabalho. Esta foi a experiência que ficou, para esses autores, da tentativa de descrever uma espécie de estado da arte desse assunto. Quaisquer informações relevantes para este fim serão muito bem-vindas.

Uma tradição que vem se mantendo e ampliando em Contabilidade é o uso de exemplos aritméticos para apresentação e explicação de conceitos e teorias contábeis. Entretanto, a natureza da Contabilidade é algébrica, como bem observou Luca Pacioli ao relacionar débitos e créditos à teoria dos números positivos e negativos. Sendo assim, a álgebra contida nas matrizes razonetes, determinada pelo Princípio das Partidas Dobradas (PPD), combinada com a lógica clássica: Princípio da Não Contradição, Princípio 
do Terceiro Excluído, cálculo de proposições, tabelas verdade, silogismo aristotélico, regras de dedução como Modus Ponens, Modus Tollens, implicações, equivalências, etc., e ainda mais, inspirada no ideal cartesiano de clareza e distinção introduzido por Renée Descartes (nascido em 31 de março de 1596, em La Haye, hoje Descartes, Touraine, França, e falecido em 11 de fevereiro de 1650, em Estocolmo, Suécia) (DESCARTES, 1637):

... a primeira regra é a evidência: não admitir "nenhuma coisa como verdadeira se não a reconheço evidentemente como tal". Em outras palavras, evitar toda "precipitação" e toda "prevenção" (preconceitos) e só ter por verdadeiro o que for claro e distinto, isto é, o que "eu não tenho a menor oportunidade de duvidar"; a segunda regra, é a da análise: "dividir cada uma das dificuldades em tantas parcelas quantas forem possíveis"; a terceira regra, é a da síntese: "concluir por ordem meus pensamentos, começando pelos objetos mais simples e mais fáceis de conhecer para, aos poucos, ascender, como que por meio de degraus, aos mais complexos"; a quarta regra é a dos "desmembramentos tão complexos... a ponto de estar certo de nada ter omitido"...

constitui o fundamento último da verdade contábil.

Um argumento não fundamentado nesta última instância não é rigorosamente contábil de acordo com o modelo formalizado por Luca Pacioli.

\section{RESULTADOS E DISCUSSÕES}

A seguir, é apresentado um exemplo de uma argumentação contábil tradicional que recorre à aritmética, mas "parece se esquecer" da álgebra, das partidas dobradas e da lógica elementar, guiadas pelo ideal cartesiano de clareza e distinção.

\section{TABLE 1 [NURNBERG, 1989; DRTINA \& LARGAY, 1985] DRTINA AND LARGAY ILLUSTRATIONS}

\section{$\underline{\text { Panel } 1 \text {-Assumptions }}$}

Schedule of Production (Physical Units)

Beginning inventory

Add: Production for period

Total available

Less: Sales for period

Ending inventory

\section{Cost per Manufactured Unit}

Variable - direct materials, direct labor, variable overhead - all out-of-pocket

Fixed - all depreciation ( $\$ 5,000 / 5,000$ units produced)

Total

Other

No change in work-in-process, receivables, or payables 
$\underline{\text { Panel } 2 \text { - Calculation of Cash Flow from Operations }}$

Direct Method

Collections (4,000 units sold @ \$ 5)

Payments (5,000 units produced @ \$ 2 variable manufacturing cost)

$\$ 20,000$

Cash flow from operations (correct)

$\underline{\$ 10,000}$

Indirect Method

Sales (4,000 units@ @ \$)

Cost of sales — LIFO (4,000 units @ \$3 full cost)

Net income

Add: Depreciation expensed in cost of sales (4,000 units @ \$ 1)

Working capital provided by operations

Less: Increase in inventory (1,000 units@ @ 3)

Cash flow from operations (incorrect)

$\$ 20,000$

$\$ 12,000$

$\$ 4,000$

\begin{tabular}{ll}
$\$ 3,000$ \\
\hline$\$ 9,000$ \\
\hline \hline
\end{tabular}

TABLE 2 [NURNBERG, 1989; DRTINA \& LARGAY, 1985]

EXTENSION OF DRTINAAND LARGAY ILLUSTRATIONS

$\underline{\text { Panel } 1 \text { - Indirect Method }}$

Drtina-Largay

Alternative

Sales (4,000 units @ \$ 5)

Cost of sales — LIFO (4,000 units @ \$3 full cost)

$\$ 20,000$

$\$ 20,000$

Net income

$\$ 12,000$

$\$ 12,000$

$\$ 8,000$

$\$ 8,000$

Add: Depreciation incurred for period (5,000 units @ \$1)

$\$ 5,000$

Add: Depreciation expensed in cost of sales (4,000 units@ @ 1)

Working capital provided by operations

Less: Increase in inventory (1,000 units@ @ 3)

Less: Increase in inventory net of depreciation capitalized therein (1,000 units@ @ 2)

$\$ 13,000 \quad \$ 12,000$

$\$ 3,000$

$\$ 2,000$

$\$ 10,000$

$\$ 10,000$

Panel 2 - Direct Method

Drtina-Largay

Alternative

Sales (4,000 units@ @ 5)

$\$ 20,000$

$\$ 20,000$

Less: Increase in receivables

Cash receipts from operations

Cost of sales - LIFO (4,000 units@ @ 3 full cost)

$\frac{-0-}{\$ 20,000}$

-0 -

$\$ 12,000$

$\$ 20,000$

$(5,000)$

$\$ 12,000$

Less: Depreciation incurred

Less: Depreciation expensed

Add: Increase in inventory (1,000 units @ \$ 3)

$\$ 3,000$

Less: Increase in inventory net of depreciation capitalized

therein (1,000 units@ @ 2)

$\$ 2,000$

Less: Increase in payables

Cash payments for production

$\frac{-0-}{\$ 10,000}$

-0 -

Cash flow from operations

$\$ 10,000$

$\$ 10,000$

$\$ 10,000$ 


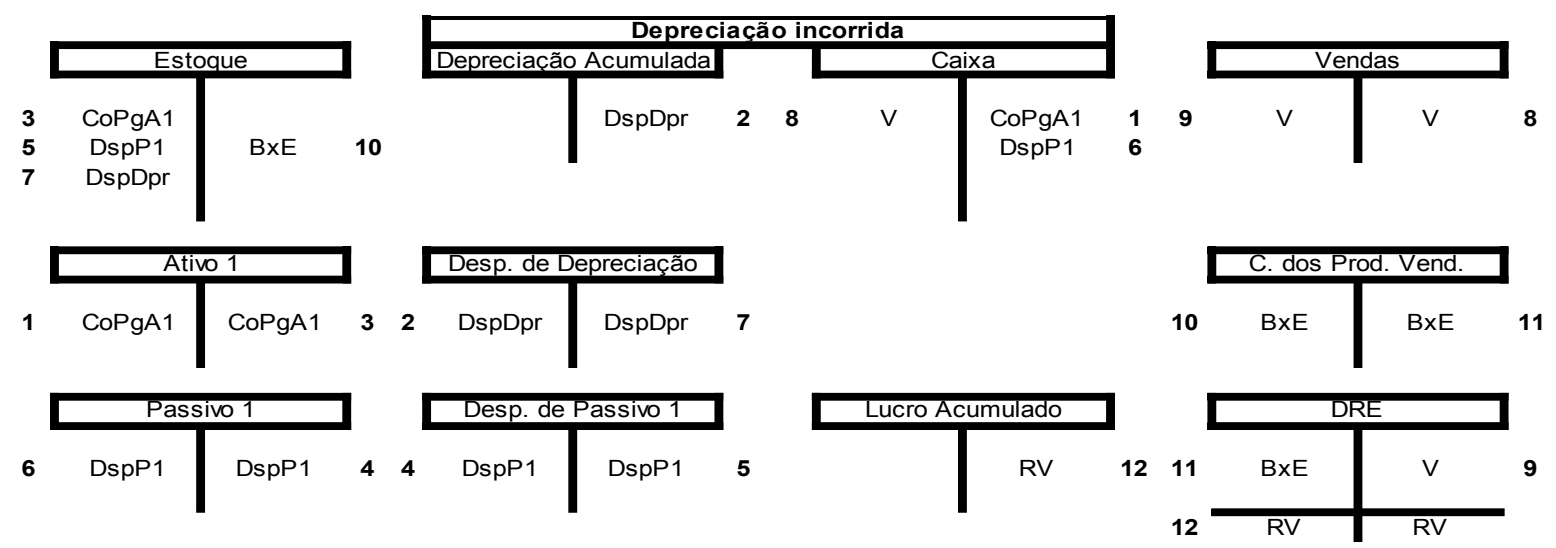

Um cenário mais coerente com a natureza algébrica deste problema de depreciação em empresas manufatureiras utiliza a álgebra implícita nas matrizes razonetes. "V" é a notação para "uma venda qualquer fixada para análise". Este é um ponto teórico sutil uma vez que "V" é variável porque indica uma venda qualquer com a propriedade de estar fixada para análise. Esta postura teórica é matematicamente superior a "considere uma venda de \$20,000". Esta última é análoga a afirmar que "a ordem das parcelas não altera a soma porque $2+3=3+2$ ". A postura algébrica afirma que "a ordem das parcelas não altera a soma porque $U+V=V+U$, quaisquer que sejam $U$ e $V$ '. Portanto, a igualdade " $2+3=3+2$ " não explica a invariância da soma por ordem das parcelas; muito pelo contrário, ela é que é explicada pela invariância da soma por ordem das parcelas. A postura algébrica cria um cenário contábil mais claro e distinto. Além disso, a notação algébrica elimina a perda de generalidade aritmética da abordagem de Nurnberg, Drtina e Largay (1993), e as matrizes razonetes permitem que se verifique rigorosamente, com clareza e distinção, se o PPD foi aplicado corretamente. Finalmente, recorrendo à lógica clássica elementar, é possível, então, rastrear os lançamentos e entender a razão de o erro apontado por Drtina e Largay ter acontecido. Estes autores atribuem o erro a uma "aplicação mecânica" do Método Indireto da DFC. Não explicam o que significa "aplicação mecânica". Uma análise algébrica esclarece o problema com a matriz acima acompanhada de roteiro lógico a seguir e da extração de [3] das parcelas envolvidas no problema.

Definicão 4.1 Uma subvariação é qualquer lançamento registrado no Livro Razão de um período. Uma variação é qualquer diferença entre saldos de uma conta de dois Balanços Patrimoniais consecutivos.

Definição 4.2 Uma partição $\wp[C]=\left\{C_{1}, C_{2}, \ldots, C_{n}\right\}$ de um conjunto $C$ é um conjunto de subconjuntos disjuntos $C_{1}, C_{2}, \ldots, C_{n}$, de $C$ tais que $C=\cup_{i} C_{i}$.

Definiç̃o 4.3 Seja $S$ um conjunto de subvariações de um período. Definiu-se:

$$
\begin{gathered}
\Delta \mathbf{E q C X}[S]=-\{\boldsymbol{\Sigma}[x \mid x \in S \text { é débito em ativo }]-\Sigma[x \mid x \in S \text { é crédito em ativo }]\} \\
+\{\Sigma[x \mid x \in S \text { é crédito em passivo }]-\Sigma[x \mid x \in S \text { é débito em passivo }]\}+ \\
+\{\Sigma[x \mid x \in S \text { é crédito em patrimônio líquido }]-\Sigma[x \mid x \in S \text { é débito em patrimônio líquido }]\}
\end{gathered}
$$

Teorema da aditividade das matrizes razonetes [TAMR] Sejam $C$ um conjunto de subvariações de um período e $\wp[C]=\left\{C_{1}, C_{2}, \ldots, C_{n}\right\}$ uma partição de $C$. Então,

$$
\Delta \mathbf{E q C x}[C]=\Sigma_{i} \Delta \mathbf{E q C x}\left[C_{i}\right] .
$$

A equação [3] é um caso particular do TAMR onde a partição máxima do conjunto das subvariações do período foi considerada, isto é, cada subvariação formou um subconjunto unitário. 
Se uma venda $\vee$ à vista ocorreu, então havia um estoque de produtos acabados disponíveis para venda. Elimina-se aqui a perda de generalidade presente no tratamento de variáveis como constantes. Uma compra de material a ser manufaturado, representada pela variável CoPgA1 do Ativo 1 (lançamento 1), foi feita à vista. Não há perda de generalidade em supor que apenas um ativo está envolvido, ou seja, matéria-prima, uma vez que índices $2,3, \ldots$, poderiam ser utilizados em outros mais, e o TAMR se aplicaria.

Na leitura do artigo de Nurnberg, no caso da depreciação incorrida, inferiu-se que o Ativo 1 depreciou-se em DspDpr (lançamento 2), e foi transformado em produto acabado (lançamentos 2, 3, 4, 5, 6 e 7). O Estoque recebeu o crédito de CoPgA1 em contrapartida com o Ativo 1, o crédito DspP1 em contrapartida com Passivo 1 - não há perda de generalidade em supor que este passivo é salário, pois novamente seria aplicado o TAMR a outros passivos indexados por $2,3, \ldots$ - e o crédito DspDpr em contrapartida com Despesa de Depreciação pela interpretação da hipótese de que a depreciação foi incorrida no período. A venda $\vee$ foi debitada no caixa (lançamento 8) em contrapartida com a conta de resultado Vendas. Para se estudar contribuição desta venda para a DFC, deve-se transferi-la, por meio de partidas dobradas, para a DRE (lançamento 9). O custo do produto vendido deve ser baixado para a DRE (lançamentos 10 e 11), e a contribuição dessa venda ao lucro operacional líquido deve ser computada e o resultado RV deve ser transferido para Lucro Acumulado (lançamento 12). Supôs-se RV positivo sem perda de generalidade porque é fácil imaginar a configuração algébrica análoga se $R V$ fosse negativo. Por sua vez, $\mathbf{\Delta E q C x}[\mathrm{V}]$ gera uma "parcela" DFC [V] de DFC. Pelo TAMR, $\mathbf{E} \mathbf{E q C x}$ é a soma de todas as parcelas $\mathbf{\Delta E q C x}$ [subvariação] geradas por todas as subvariações do período relativas a $\vee$. Extraindo-se de [3] apenas o conjunto $C$ de subvariações envolvidas com $\vee$, obtém-se:

$\Delta \mathrm{EqCx}[C]=-\Sigma \Delta$ [c. de at. de $C]+\Sigma \Delta$ [c. de pass. de $C]+\Sigma \Delta$ [c. de p. I.]

$\Delta \operatorname{EqCx}[C]=\Delta[$ Estoque $[C]]+\Delta[$ Depreciação Acumulada $[C]]+\Delta[$ Lucro Acumulado $[C]]$

$\Delta \mathbf{E q C x}[C]=-[C O P g A 1+D s p P 1+D s p D p r-B x E]-[-D s p D p r]+[\quad+R V]$

Ativo 1 e Passivo 1 possuem variação $\Delta=0$, relativa a $\vee$, e não precisam ser explicitados nessa demonstração.

$\Delta \mathbf{E q C x}[C]=-[C O P g A 1+D s p P 1+D s p D p r-B \times E]-[-D s p D p r]+[+V-B \times E]$

$\Delta \mathbf{E q C x}[C]=V-[C O P g A 1+D s p P 1]$

A álgebra oferece cancelamentos deixando apenas recebimentos e pagamentos! Ou seja, ela deixa uma parcela $\mathrm{DFC}[\mathrm{V}]$, relativa à venda $\vee$ e correspondente às atividades operacionais relativas à venda $\checkmark$, pelo Método Direto! Entretanto, se se examinar com um pouco mais de atenção as equações acima, todas equivalentes à relação invariante $[3][C]$, pode-se reescrevê-la em mais duas formas equivalentes:

$$
\begin{aligned}
& \Delta \mathbf{E q C x}[C]=[V-B \times E]-[-D s p D p r]-[\operatorname{CoPgA} 1+D \operatorname{spP} 1+D s p D p r-B \times E] \\
& \Delta \mathbf{E q C x}[C]=L L O p[C]+D s p D p r[C]-\Delta \text { Estoque }[C]
\end{aligned}
$$

A álgebra deu agora a conciliação do lucro operacional líquido LLOp[C] com o EqCx! Ela é generosa, dá mais do que se lhe pede: agora foi obtida a parcela $\mathrm{DFC}[C]$, correspondente às atividades operacionais relativas à venda V, pelo Método Indireto! É importante observar que a DFC $[C]$ "bateu" porque a álgebra demonstra este fato com clareza e distinção, e tem-se "certeza" de que a DFC $[C]$ está certa, podendo-se conferir a sua dedução quantas vezes se quiser. Além disso, se houver erros, podem-se percorrer cuidadosamente os passos dedutivos e descobrir onde eles estão, e corrigi-los.

$\mathrm{Na}$ análise de Nurnberg e Drtina e Largay, fica claro o significado de "algoritmo algébrico para a DFC". A obtenção simultânea dos dois Métodos não foi uma coincidência como se mostrará adiante. O leitor pode aplicar algoritmo sugerido para a DFC [V] examinando o caso (considerando a matriz análoga) em que Nurnberg supõe que a depreciação é realizada apenas com a venda. 
O algoritmo expressa um invariante algébrico contábil ( $\Delta \mathbf{E q C X})$ em uma sequência de formas equivalentes. Utiliza-se a notação algébrica a seguir para indicar os elementos do conjunto $\Delta \Delta \mathrm{CONTA}$, denominados subvariacões, agrupados nos conjuntos denotados por $\Delta C O N T A$ denominados variacões. Tais subvariações são as variáveis algébricas. Subvariações são as variáveis que assumem os valores dos lançamentos. Logicamente, subvariação é variável e lançamento é constante, do mesmo modo que $x$ é variável e 1 é constante que pode ser substituída em $x$. A seguir, são definidas as variáveis de um exemplo de DFC (observe-se que este exemplo é algébrico e, portanto, livre da particularidade numérica presente, por exemplo, no Manual (FIPECAFI 2010)).

\section{$\triangle \triangle$ CONTA}

AprDspa

AumC

BImb

Blnc

CMV

Co

$D P R$

DSCD

DSPDV

DspDpr

DspF

DSPPDD

DspS

DDvd

IR

LAIR

$L B$

LL

LVImb

NE

Prcld

PgDvd

$P g E$

PgF

PgIR

PgS

PrVIR

$R D P$

RecF

$\checkmark$ apropriação de despesa antecipada

aumento de capital

baixa de imobilizado

baixa de valor incobrável

custo da mercadoria vendida

compras

duplicatas a receber

desconto de duplicata

despesas diversas

despesa de depreciação

despesa financeira

despesa de PDD

despesa de salários

dividendo distribuído

imposto de renda

lucro antes do IR

lucro bruto

lucro líquido

lucro na venda de imobilizado

novo empréstimo

provisão para crédito de liquidação duvidosa

pagamento de dividendo

pagamento de empréstimo

pagamento de fornecedor

pagamento de IR

pagamento de salário

provisão para IR

recebimento de duplicatas

receita financeira

vendas

$\Delta \Delta$ CONTA
$\Delta \mathrm{DspA}$
$\Delta \mathrm{C}$
$\Delta \mathrm{Imb}$
$\Delta \mathrm{DRec}$
$\Delta \mathrm{E}$
$\Delta \mathrm{F}, \Delta \mathrm{E}$
$\Delta \mathrm{DRec}$
$\Delta \mathrm{DscD}$
$\Delta \mathrm{DspDv}$
$\Delta \mathrm{DspDpr}$
$\Delta \mathrm{DspF}$
$\Delta \mathrm{DspPDD}$
$\Delta \mathrm{DspS}$
$\Delta \mathrm{DDvd}$
$\Delta \mathrm{IR}$

$\Delta$ Lac

$\Delta$ Empr

$\triangle P D D$

$\Delta$ Lac, $\Delta$ DDvd

$\Delta$ Empr

$\Delta \mathbf{F}$

$\Delta \mathrm{IR}$

$\Delta \mathrm{S}$

$\Delta \mathrm{IR}$

$\Delta$ DRec

$\Delta \mathbf{R F}$

$\Delta$ DRec

Em alguns períodos, podem ser zero e em outros são acompanhadas de novas subvariações que não foram relacionadas acima. Poderão aparecer sempre no papel de trabalho apresentado abaixo e ser anuladas quando forem zero no período considerado, sem prejuízo algum para a elaboração da DFC. Separando as contas, de acordo com o CPC 03, nas categorias de Atividades Operacionais, Atividades de Investimento e Atividades de Financiamento, pode-se expressar $\mathbf{\Delta E q C x}$ como a soma de variações: 
$(\Delta \mathrm{DRec})+(\Delta \mathrm{DDsc})+(\Delta \mathrm{PCLD})+(\Delta \mathrm{E})+(\Delta \mathrm{DspA})+\Delta \mathrm{F}+\Delta \mathrm{IR}+\Delta \mathrm{S}+$

$(\Delta \mathrm{Imb})+(\Delta \mathrm{DprAc})+$

$\Delta \mathbf{E m p r}+\Delta \mathrm{C}+\Delta \mathbf{L a c}=$

que equivale, respectivamente, à soma de subvariações:

$$
\begin{aligned}
& =-[V-B i n c-R D p]-[-D s c D]-[B \operatorname{lnc}-D \text { spPDD }]-[C o+C M V]-[D \text { spA }- \text { AprDspA }] \\
& +\left[-P g F+C_{0}\right]+[-P g I R+P r v I R]+[-P g S+D s p S]+ \\
& -[A q l m b-B l m b]-[B D p r-D s p D p r]+ \\
& {[-P g D \text { spF }+D \text { spF }+N E]+[\text { Aum C + }]+[- \text { PgDrd }+L L]=}
\end{aligned}
$$

LLé transportado para o início da linha e é substituído pela soma equivalente a ele dada pela DRE, destacando-se as parcelas que se cancelarão:

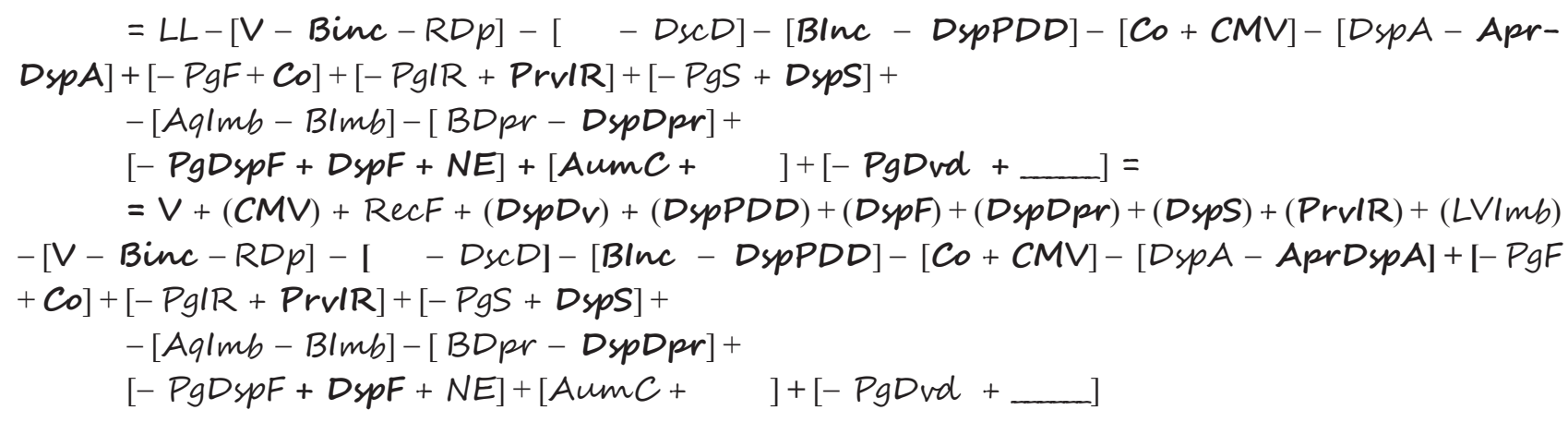

Definiu-se, precisamente, uma subvariação de efeito líquido nulo em $\mathbf{\Delta E q C x}$ como sendo qualquer uma que comparece nessa soma juntamente com seu oposto aditivo. Portanto, fica definida, logicamente, uma subvariação de efeito líquido não nulo em $\Delta \mathbf{E q C X}$ como sendo qualquer uma que não possui seu oposto aditivo nessa soma. A palavra "líquido" dá conta do possível anulamento do efeito sobre $\Delta \mathbf{E q C x}$. "Anulamento" aqui é apenas uma propriedade algébrica, não é o mesmo que "desaparecimento". A equação [3] mostra claramente que qualquer lançamento transforma-se em subvariação que tem efeito

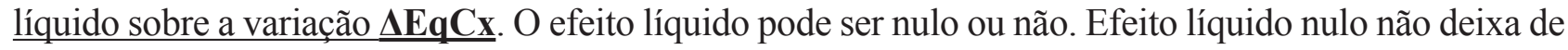
ser efeito. Aálgebra permite tratar igualmente todos os lançamentos como parcelas de uma mesma equação. O mais importante é que todos os efeitos, isto é, todas as subvariações ou lançamentos do período, estão sob absoluto controle algébrico de quem elabora a DFC.

É importante notar que, uma vez que o estudante possua a lista de subvariações das contas da empresa, o problema de trabalhar na confecção da DFC passa a ser puramente algébrico. Ele tem a equação [3], e formas equivalentes dela, o tempo todo em que trabalha na DFC, e nunca perde de vista essa igualdade deduzida dos dois balanços consecutivos desde o início. Computam-se todos os cancelamentos, exceto os que envolvem o lucro na venda de imobilizado:

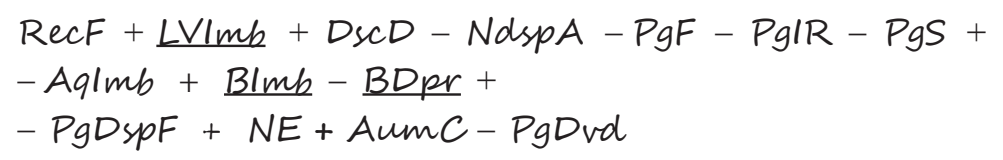

Por definição, uma subvariação como -BDpr, pelo fato de não estar presente juntamente com seu

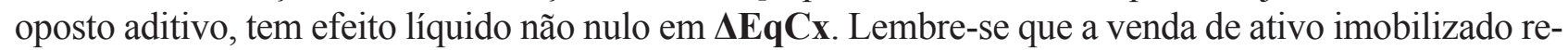
laciona subvariações por meio da seguinte equação: $L V I m b=V I m b+B D p r-B i m b$. Logo, a expressão equivalente de $\Delta \mathbf{E q C x}$ se torna: 


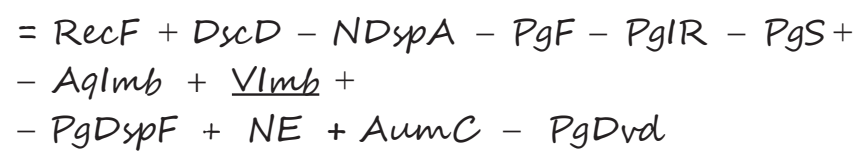

É um fato algébrico - consequência das partidas dobradas - o cancelamento, nessa expressão, das subvariações de efeito líquido nulo sobre o Equivalente de Caixa, restando aquelas que se constituíram em pagamentos e recebimentos no período e mais algumas, aparentemente criando dificuldades para o modelo. Entretanto, surge uma situação interessante. Existem subvariações [LVImb, BDpr e Blmb] cuja soma [Vimb] é um recebimento (poderia ser um pagamento). Pela equação [3], toda subvariação que não foi cancelada está associada a outras que não são pagamentos, nem recebimentos, cuja soma é a mesma de pagamentos e recebimentos. Uma subvariação que não foi cancelada não pode estar sozinha no segundo membro de [3].

Teorema 4.4 [Teorema da DFC direta] Toda subvariação da equação variacional fundamental, que não seja pagamento nem recebimento, não cancelada pela presença de sua contrapartida dobrada, pode ser associada a um conjunto não vazio de outras subvariações cuja soma seja zero ou uma soma de pagamentos e recebimentos.

O contador possui os documentos necessários para identificar os pagamentos e recebimentos que, somados, se igualam a somas de subvariações. No exemplo, o contador identifica as subvariações LVImb, Blmb e - BDpr com o recebimento $V I m b$. A álgebra oferece a ocorrência de situações onde vários pagamentos e recebimentos são somas de subvariações que ficaram no segundo membro, assim como uma situação em que essas subvariações nunca se agrupam para formar um pagamento ou um recebimento em particular. É interessante saber se esses exemplos existem em Contabilidade. Se não existirem, então não há nenhum problema lógico, a não ser o fato de que a álgebra frequentemente dá mais do que se lhe pede.

Não é exagerado enfatizar que o tratamento algébrico das subvariações descreve rigorosamente, e naturalmente, a demonstração da DFC pelo Método Direto. Por esse caminho, o estudante não tem mais pretexto para exclamar: “... não sei por onde começar!”. Além disso, se começar, então sempre saberá se está indo bem e se terminará bem! A álgebra lhe oferece uma visão clara e distinta, que lhe permite controle total, de todos os lançamentos registrados no período. Uma sequência de igualdades o conduz até o último elemento da DFC que é o par de matrizes contendo o método direto e o indireto.

Há duas representações de DFC concebíveis. Uma é a algébrica, que só contém subvariações das contas que podem ser as mesmas durante um bom tempo, pelo menos enquanto o plano de contas não seja alterado. A outra é a DFC aritmética, que consiste na substituição da DFC algébrica pelas constantes que representam os lançamentos que geraram o segundo balanço. Portanto, mais uma vez, confirma-se a afirmação de D'Alembert. A DFC algébrica pode ser feita uma vez e fornecer várias DFCs aritméticas por meio de uma mera substituição de variáveis por constantes. Podem ocorrer mais ou menos subvariações, mas nas mesmas contas.

Para se derivar um teorema para o Método Indireto da DFC, análogo ao Teorema 4.4, é preciso uma matriz $m \times n$ e uma estrutura de submatrizes. Para o Método Direto, bastou uma matriz $1 \times 1$, isto é, uma variável, a saber, $\Delta \mathbf{E q C X x}$, e uma sequência de expressões dessa variável para se gerar a DFC. A necessidade de matrizes com linhas e colunas para tratar a álgebra do Método Indireto é um indicador claro da maior dificuldade envolvida nesse demonstrativo por meio de uma sequência de "matrizes equivalentes" onde a última é exatamente a DFC pelo Método Indireto. A matriz denominada "DFC" possui uma estrutura que acomoda, em suas submatrizes, entradas para o registro dos valores que se referem a Atividades Operacionais (Matriz ATVOP I, Matriz ATVOP D), Atividades de Investimento (Matrizes ATVINV e ATVINV') e Atividades de Financiamento (Matrizes ATVF e ATVF'), além da Matriz DRE, da Matriz $\Delta \mathbf{B P}$, da Matriz $\Delta \Delta \mathbf{B P}$ e da Matriz AJLL.

Pode-se usar a matriz abaixo como papel de trabalho para os leitores anotarem os valores de acordo com as definições de DFC pelo CPC 3. Note-se que uma submatriz, denominada $\triangle B P$, está imersa, em DFC, contendo como entradas as variações das contas relacionadas na equação equivalente a [3]: 


$$
\begin{gathered}
(\Delta \mathrm{DRec})+(\Delta \mathrm{DDsc})+(\Delta \mathrm{PCLD})+(\Delta \mathrm{E})+(\Delta \mathrm{DspA})+\Delta \mathrm{F}+\Delta \mathrm{IR}+ \\
+\Delta \mathrm{S}+(\Delta \mathrm{Imb})+(\Delta \mathrm{DprAc})+\Delta \mathrm{Empr}+\Delta \mathrm{C}+\Delta \mathrm{Lac}=\Delta \mathrm{EqCx}
\end{gathered}
$$

Portanto, a coluna das variações $\Delta$ CONTA com sinal adequado tem soma $\mathbf{\Delta E q C x}$. A matriz $\triangle E Q C X$ é justaposta abaixo da matriz DFC para registrar o cálculo direto da variação da conta Equivalente a Caixa denotada por EqCx. O preenchimento de $\Delta \mathbf{B P}$ é o primeiro de uma sequência que representa o invariante $\Delta \mathbf{E q C x}$ expresso em formas equivalentes a [2]. Este é o ponto de partida do algoritmo prático para DFC.

Definicão 4.5 Dados dois balanços consecutivos, a matriz diferencial $\Delta$ BP é a matriz $n \times 2$ contendo as variações das contas no período considerado. O número $n$ de linhas é um número suficiente para abrigar todas as contas separadas nas matrizes ATVOP I, ATVOP D, DRE, ATVINV e ATVFIN como requisitado pelo CPC 3. A matriz-coluna $\mathbf{\Delta} \mathbf{\Delta B P}$ abriga as subvariações que afetaram as contas no período considerado. Automaticamente, $\Delta \mathbf{E q C x}$ é a soma da coluna da direita. O ponto de partida nessa sequência é a igualdade

$$
\begin{aligned}
\Delta \mathrm{EqCx}= & (\Delta \mathrm{DRec})+(\Delta \mathrm{DDsc})+(\Delta \mathrm{PCLD})+(\Delta \mathrm{E})+(\Delta \mathrm{DspA})+\Delta \mathrm{F}+\Delta \mathrm{IR}+ \\
& +\Delta \mathrm{S}+(\Delta \mathrm{Imb})+(\Delta \mathrm{DprAc})+\Delta \mathrm{Empr}+\Delta \mathrm{C}+\Delta \mathrm{Lac}
\end{aligned}
$$

Ela é exatamente a primeira equivalência do algoritmo, deduzida do segundo invariante fundamental $\Delta \mathbf{A}=\boldsymbol{\Delta} \mathbf{P}+\boldsymbol{\Delta} \mathbf{P L}$ da Contabilidade. Na coluna da esquerda, isto é, do lado esquerdo das variações algébricas, registram-se as variações aritméticas das contas. Na coluna da direita, isto é, na matriz-coluna $\mathbf{\Delta} \mathbf{\Delta B P}$, registram-se algebricamente as subvariações deduzidas do CPC 3. A matriz $\mathbf{\Delta E Q C X}$ registra a confirmação do invariante $\Delta \mathbf{E q C X}$. As matrizes acima são equivalentes no sentido de que suas linhas são equivalentes e, portanto, a soma de suas linhas é a mesma, exatamente igual $\mathbf{\Delta E q C x}$, o valor da DFC que precisa ser "demonstrado". O algoritmo irá manter a "certeza" de que a DFC "vai bater", porque a sequência sempre produz a soma $\Delta \mathbf{E q C X}$ e, portanto, se a última matriz satisfizer à definição de DFC do CPC 3, então a DFC estará correta e "terá batido". Além disso, o algoritmo permite tantas verificações quantas forem necessárias para que o leitor se "convença" de que a DFC produzida está certa. A mais importante característica desse algoritmo é que ele permite ao leitor procurar eficientemente a origem de eventuais erros como, por exemplo, a não obtenção da soma $\Delta \mathbf{E q C x}$ em qualquer dos passos, e não apenas no último que produz a DFC.

O passo anterior mostra claramente qual é a "intenção" do algoritmo e porque ele é denominado "algébrico". O LL é retirado da linha $\Delta \mathbf{L a c}$ e transportado para a última linha da matriz AJLL, sem sair da matriz ATVOP I. Portanto, se as linhas da coluna da direita dessa matriz forem somadas, tomando-se o cuidado de incluir a subvariação - Dvd que foi deslocada para a coluna à sua direita, a soma $\mathbf{\Delta E q C x}$ continuará sendo obtida. A variação $\Delta$ Lac não aparece mais em ATVOP I, mas continua a contribuir igualmente para a soma das linhas da coluna que é exatamente a variação $\Delta \mathbf{E q C x}$ da conta Equivalente de Caixa. A matriz ATVOP D foi alterada, deixou de ser vazia. Ela contém agora as subvariações LLe $D v d$. A soma de sua coluna esquerda é $\Delta \mathbf{L a c}=L L-D v d$. Portanto, ATVOP D começou a ser preenchida com as subvariações de $\mathbf{\Delta} \mathbf{\Delta B P}$ e, quando todas as subvariações forem transportadas para ela, a soma de suas linhas será, evidentemente, $\mathbf{\Delta E q C x}$. O algoritmo irá manter em ATVOP D apenas as subvariações pagamentos e recebimentos. Logo, LL não pode permanecer nessa matriz uma vez que não é esse tipo de lançamento. O algoritmo, então, substitui LL pelas subvariações da DRE cuja soma equivale a ele. Isto é, as subvariações que produziram a DRE são introduzidas para fornecer um valor equivalente ao LL.

Excluem-se, então, as linhas somas da DRE, ficando apenas com as subvariações parcelas. A matriz ATVOP D foi transformada em uma matriz equivalente no sentido de que a soma de suas linhas continua a resultar em $\Delta \mathbf{L a c}=L L-D v d$. Ela contém agora as subvariações equivalentes à $L L$. Como subvariações que não sejam pagamentos ou recebimentos não podem permanecer nessa matriz, o algoritmo, para ATVOP I, obedecendo às definições de DFC do CPC 3. 
Como exemplo, observe-se o transporte das subvariações $C M V$ e $-V$ para ATVOP D, zerando suas posições em $\mathbf{\Delta} \mathbf{\Delta B P}$. As variações $-\mathbf{\Delta D R e c}$ e $-\mathbf{\Delta E}$ não são alteradas, de modo que a soma das linhas em ATVOP I continua invariante. Quanto à matriz ATVOP D, duas parcelas novas dão entrada em suas linhas, mas cancelam seus opostos aditivos que estão nas linhas da submatriz DRE. Esse cancelamento foi totalmente benéfico uma vez que tais subvariações não podem permanecer em ATVOP D pelo fato de não serem nem pagamentos nem recebimentos. - Co e Co podem ser excluídos das linhas de $\mathbf{\Delta} \mathbf{\Delta} \mathbf{B P}$ uma vez que se cancelam na soma dessas linhas. Outros cancelamentos análogos são possíveis, mas o algoritmo deve ser explicado devagar para facilitar o entendimento do leitor. Ao transportarmos -PgS + DspS para ATVOP D, DspS cancela com (DspS) e elimina uma subvariação que não afetou diretamente a conta Equivalente a Caixa. Assim, a matriz ATVOP D recebe mais duas subvariações de $\mathbf{\Delta B P}$, mantém sua característica de conter apenas pagamentos e recebimentos do período, e segue a sequência de transformações que a levarão à demonstração da DFC pelo método direto.

Como controle, há a matriz ATVOP I onde se mantém a variação $\Delta \mathbf{S}$ que se iguala ao valor -PgS + DspS cujas parcelas foram transportadas para ATVOPD. O leitor pode observar facilmente que é equivalente considerar e manter a variação $\Delta S$ em ATVOP I e introduzir o pagamento -PgS em ATVOPD. Na verdade, a parcela $D s p S$ acompanha esse pagamento a fim de que se preserve a variação $\Delta \mathbf{S}$, mas a álgebra se encarrega de cancelá-la com seu oposto aditivo (DspS) que havia entrado em ATVOP D junto com as subvariações da DRE que substituíam o LL. Esse pequeno "milagre" lembra a afirmação de D'Alembert sobre a álgebra. Portanto, o invariante $\mathbf{\Delta E q C x}$ não se altera, enquanto soma das linhas da matriz ATVOP I, devido a essa operação de eliminar duas subvariações de $\mathbf{\Delta} \mathbf{\Delta} \mathbf{B P}$ dando um passo na direção da formação da DFC pelo método direto. $O$ transporte de $-P g I R+\mathbb{R}$, para a matriz ATVOPD, pode ser descrito de maneira exatamente análoga à $-P g S+D s p S$. Como controle, tem-se a matriz ATVOP I onde a variação $\Delta I R$ é mantida e se iguala ao valor $-P g I R+I R$, cujas parcelas foram transportadas para ATVOPD. É equivalente considerar e manter a variação $\triangle I R$ em ATVOP I e introduzir o pagamento - PgIR em ATVOPD. Na verdade, a parcela IR acompanha esse pagamento a fim de que se preserve a variação $\Delta \mathbf{I R}$, mas a álgebra se encarrega de cancelá-la com seu oposto aditivo (IR) que havia entrado em ATVOP D junto com as subvariações da DRE que substituíam o LL.

O transporte de $-P D s p F+N E+D s p F$ para a matriz ATVOP D permite que se apague $\Delta$ Empr em ATVOP I. Entretanto, como -PDspF deverá pertencer a ATVOP I por convenção do CPC 3, esta subvariação sobe para se agrupar com LL em ATVOP I e sobe em ATVOP D para que a soma de atividades operacionais coincida nos dois métodos. O transporte de -Aqlmb + Blmb para a matriz ATVOP D permite que se apague $\Delta \mathbf{I m b}$ em ATVOP I. Entretanto, como BImb deverá compor a Vimb que ficará na mesma linha de -AqImb em ATVOP D, BImbé cancelada dando lugar a Vimb. Para se recuperar a equivalência das matrizes afetada pela entrada de Vimb em ATVOP D, introduz-se ( Lvimb) em ATVOP I agrupada a LLe cancelam-se Lvimb, -BDpr e DspDpr em ATVOPD, sendo que esta última implica em apagar $\Delta$ DspDpr. O transporte de AumC para a matriz ATVOP D permite que se apague $\Delta$ C em ATVOP I.

\section{CONCLUSÕES}

Esta pesquisa de natureza normativa concluiu seu objetivo de contribuir para fechar a lacuna de um método claro, seguro e efetivo para a DFC. Ofereceu um algoritmo para a elaboração da DFC e baseou-se nas relações algébricas entre variáveis contábeis dispostas em matrizes. Como resultado, seguiu-se o Algoritmo para a DFC, disposto em dados e seis passos.

Dados: São dadas $\boldsymbol{m}$ contas de ativo classificadas como operacionais. São dadas $\boldsymbol{n}$ contas de passivo classificadas como operacionais. São dadas $\boldsymbol{r}$ contas de ativo classificadas como investimentos. São dadas $\boldsymbol{s}$ contas de patrimônio líquido classificadas como financiamentos.

Passo 1: ATVOP I é preenchida conforme o modelo apresentado pelas variações $\Delta_{i}$.

Passo 2: $\Delta \Delta$ BP é preenchida pelas subvariações $\Delta \Delta_{i, k}$ que compõem as variações $\Delta_{i}$.

Passo 3: A subvariação LL é transportada de $\Delta$ Lac para AJLLOp e sua expressão equivalente dada pela DRE é introduzida em ATVOP D. 
Passo 4: As subvariações $\Delta \boldsymbol{\Delta}_{i, k}$ não operacionais, que são pagamentos ou recebimentos, são transportadas para ATVINV' e ATVFIN'.

Passo 5: Aquelas subvariações que não são pagamentos ou recebimentos, não se cancelam e não são operacionais, são transportadas para ATVINV' e ATVFIN', agrupam-se de modo equivalente a pagamentos ou recebimentos e são substituídas por essas novas variáveis pagamentos ou recebimentos.

Passo 6: Aquelas subvariações que não são pagamentos nem recebimentos, e não se cancelam, se forem operacionais são transportadas para ATVOP I, agrupam-se com o LL, e seus respectivos $\boldsymbol{\Delta}_{i}$ são excluídos em ATVOP I.

Teorema da DFC: O algoritmo para a DFC é logicamente consistente, eficiente e eficaz para gerar o Método Direto e o Método Indireto simultaneamente e equivalentemente.

\section{REFERÊNCIAS}

BEINHOCKER, Eric D. The Origin of Wealth: Evolution, Complexity, and the Radical Remaking of Economics. Boston: Harvard Business School Press, 2006.

CAMPOS, Filho, Ademar. Demonstração dos Fluxos de Caixa. $1^{\text {a }}$ edição. São Paulo: Atlas, 1999.

COMITÊ DE PRONUNCIAMENTOS CONTÁBEIS - Disponível em http://www.cpc.org.br. Acesso em: fev/2010.

COMITÊ DE PRONUNCIAMENTOS CONTÁBEIS. CPC 03. Disponível em: www.cpc.org.br/pdf/ cpc03.pdf. Acesso em: 15/05/2010.

CVM - COMISSÃO DE VALORES MOBILIÁRIOS. CVM N. 547/2008. Disponível em: www.cvm. gov.br/port/infos/deli547.pdf. Acesso em: 15/05/2010.

DECHOW, Patricia. Accounting Earnings and Cash flows as Measures of Firm Performance: The Role of Accounting Accruals. Journal of Accounting and Economics, 18, 3-42. New York, Elsevier: 1994.

DECHOW, Patricia, KHOTARI, S.P., WATTS, R. L. The Correlation Structure of Earnings, Cash Flows, and Accruals. Journal of Accounting and Economics, 131-168. New York, Elsevier: 1998.

DESCARTES, Renée. Discours de la méthod pour bien conduire sa raison et chercher la vérité dans les sciences. Leiden: 1637.

DRTINA, Ralph E., LARGAY, James A., III. Pitfalls in Calculating Cash Flow from Operations. The Accounting Review, v. 60, n. 2; p. 314, Apr 1985.

FASB - FINANCIAL ACCOUNTING STANDARDS BOARD. Statement of Financial Accounting Standards n. 95 - Statement of Cash Flows. Financial Accounting Series. Financial Accounting Standards Board of the Financial Accounting Foundation. New York: November 1987.

FASB - FINANCIAL ACCOUNTING STANDARDS BOARD. International Accounting Standard - IAS 07 - Cash Flow Statements. Disponível em: www.bcb.gov.br/?IAS7. Acesso em: 10/03/2010.

FIPECAFI. Manual de Contabilidade Societária: Aplicável a todas as sociedades. 1a. ed., São Paulo: Atlas, 2010. 
GELONEZE-NETO, Antonio; KASSAI, José Roberto. Demonstração de fluxos de caixa (DFC): reflexões por meio de um algoritmo algébrico. In: CONGRESSO BRASILEIRO DE CUSTOS, XVII, 2010, Belo Horizonte/MG. Anais... Belo Horizonte: UFMG, 2010. CD-ROM.

KASSAI, José Roberto. O caso da Cia Sudoku - como elaborar a DFC - Apostila da disciplina EAC 202 Análises das Demonstrações Contábeis. FEA/USP: 2009.

LEI 6.404 de 15/12/1976. Disponível em: http:/www.planalto.gov.br/ccivil_03/leis/16404consol.htm. Acesso em: 15/03/2010.

LEI 11.638 de 28/12/2007. Disponível em http://www.planalto.gov.br/CCIVIL/_Ato2007-2010/2007/ Lei/L11638.htm. Acesso em: 15/03/2010

MACHALE, D. Comic Sections: Book of Mathematical Jokes, Humour, Wit and Wisdom. Dublin: Boole Press, 1993.

MARQUES, José Augusto V. da C., CARNEIRO, João B. A. Jr., KUHL, Carlos Alberto. Demonstração de Fluxos de Caixa. Rio de Janeiro: Freitas Bastos, 2008.

NURNBERG, H.. Inconsistencies and Ambiguities in Cash Flow Statements Under FASB Statement $\mathrm{n}$. 95. Accounting Horizons, v. 7, n. 2. New York: 1993.

NURNBERG, H.. Depreciation in the Cash Flow Statement of Manufactoring Firms: Amount Incurred or Amount Expensed? Accounting Horizons. V. 3, March 1989, p. 95-101.

PACIOLI, Luca. Summa de arithmetica, geometria, proportioni e proportionalita. Veneza: 1494.

\section{APÊNDICES}

Demonstração do TAMR: temos: $x \in C \Leftrightarrow x \in \cup_{i} C_{i} \Leftrightarrow x \in C_{1}$ ou $x \in C_{2}$ ou ... ou $x \in C_{n}$. Portanto, separando todas as subvariações de $C$ que são de ativo, passivo ou de patrimônio líquido e estão em $C_{i}$, pela propriedade associativa da adição, tem-se:

$$
\begin{gathered}
\Sigma_{\iota} \Delta \mathbf{E q C X}\left[C_{i}\right]= \\
=\left\{-\left[\Sigma a_{1}-\Sigma b_{1}\right]+\left[\Sigma c_{1}-\Sigma d_{1}\right]+\left[\Sigma e_{1}-\Sigma f_{1}\right]\right\}+\ldots+\left\{-\left[\Sigma a_{n}-\Sigma b_{n}\right]+\left[\Sigma c_{n}-\Sigma d_{n}\right]+\left[\Sigma e_{n}-\Sigma f_{n}\right]\right\}= \\
=-\left\{\left[\Sigma a_{1}+\ldots+\Sigma a_{n}\right]-\left[\Sigma b_{1}+\ldots+\Sigma b_{n}\right]\right\}+\left\{\left[\Sigma c_{1}+\ldots+\Sigma c_{n}\right]-\left[\Sigma d_{1}+\ldots+\Sigma d_{n}\right]\right\}+\left\{\left[\Sigma e_{1}+\ldots+\Sigma e_{n}\right]-\left[\Sigma f_{1}+\ldots+\Sigma f_{n}\right]\right\}= \\
=-\left\{\Sigma\left[a_{1}+\ldots+a_{n}\right]-\Sigma\left[b_{1}+\ldots+b_{n}\right]\right\}+\left\{\Sigma\left[c_{1}+\ldots+c_{n}\right]-\Sigma\left[d_{1}+\ldots+d_{n}\right]\right\}+\left\{\Sigma\left[e_{1}+\ldots+e_{n}\right]-\Sigma\left[f_{1}+\ldots+\Sigma f_{n}\right]\right\}= \\
=-\{\Sigma[x \mid x \in C \text { é déb. at. }]-\Sigma[x \mid x \in C \text { é créd. at. }]\}+\{\Sigma[x \mid x \in \text { é créd. pass. }]-\Sigma[x \mid x \in S \text { é déb. ativo }]\}+ \\
+\{\Sigma[x \mid x \in S \text { é créd. p. 1. }]-\Sigma[x \mid x \in S \text { é déb. p. } 1 .]\}=\Delta \mathbf{E q C x}[C]
\end{gathered}
$$

onde $a_{1}=$ déb. at. de $C_{1}, b_{1}=$ créd. at. de $C_{1}, c_{1}=$ créd. pass. de $C_{1}, d_{1}=$ déb. pass. de $C_{1}, e_{1}=$ créd. pl. de $C_{1}, f_{1}=$ déb. pl. de $C_{1}$.

Demonstração do Teorema da DFC Direta: Na equação [3], transportam-se para o primeiro membro todas as subvariações do segundo membro que são pagamentos ou recebimentos. Se não restou nenhuma no segundo membro, então não há nada a demonstrar e o teorema é verdadeiro. Suponha-se que o novo primeiro membro não seja zero. Então, há pelo menos duas subvariações no segundo membro. De fato, se houvesse apenas uma, ela se igualaria ao saldo de caixa do primeiro membro e, portanto, seria 


\section{repec}

obrigada a ser o pagamento ou recebimento que está faltando no fluxo de caixa, o que não é possível porque não há mais pagamentos ou recebimentos no segundo membro. Então, as subvariações não canceladas do segundo membro somam o mesmo valor do primeiro membro que é um saldo não nulo do caixa. Isso significa que há pagamentos e recebimentos cuja soma é o segundo membro. Na pior das hipóteses, todos os pagamentos e recebimentos do primeiro membro satisfazem a segunda afirmação da tese do teorema.

Demonstração do Teorema da DFC:

No Passo 1, a soma das linhas de ATVOP I é $\Delta \mathbf{E q C x}$, ou seja,

$$
\left[-\Delta_{1}-\ldots-\Delta_{m}+\Delta_{m+1}+\ldots+\Delta_{m+n}\right]+\left[-\Delta_{m+n+1}-\ldots-\Delta_{m+n+r}\right]+\left[\Delta_{m+n+r+1}+\ldots+\Delta_{m+n+r+s}\right]
$$

de acordo com o invariante [2]. No Passo 2, $\Delta \mathbf{\Delta B P}$ é preenchida pelas subvariações $\Delta \Delta_{i, k}$ que compõem as variações $\Delta_{i}$ e, portanto, a soma de suas linhas também é $\mathbf{\Delta E q C x}$. No Passo 3, a subvariação LL é transportada de $\Delta$ Lac para AJLLOp e sua expressão equivalente dada pela DRE é introduzida em ATVOP D. Portanto, a soma das linhas de ATVOP I continua invariante e ATVOP D começa a ser preenchida com as subvariações de $\Delta \mathbf{\Delta} \mathbf{B P}$, processo que culminará com o transporte de todas as subvariações de $\mathbf{\Delta} \mathbf{\Delta B P}$ para ATVOP D permanecendo somente os pagamentos e recebimentos após cancelamentos permitidos pelas PD. No Passo 4, as subvariações $\boldsymbol{\Delta} \boldsymbol{\Delta}_{i, k}$ de $\boldsymbol{\Delta} \mathbf{\Delta} \mathbf{B P}$ que forem pagamentos ou recebimentos, não operacionais, são transportadas para $\mathbf{A T V I N V}{ }^{i, k}$ e ATVFIN'. Esta ação dá prosseguimento ao processo de preencher ATVOP D, a matriz que resultará no Método Direto da DFC. No Passo 5, aquelas subvariações que não são pagamentos nem recebimentos, não se cancelam e não são operacionais, são transportadas para ATVINV' e ATVFIN', agrupam-se de modo equivalente a pagamentos e recebimentos e são substituídas por essas novas variáveis pagamentos e recebimentos, continuando a aproximar ATVOP D da DFC pelo Método Direto. No Passo 6, aquelas subvariações $\boldsymbol{\Delta} \boldsymbol{\Delta}_{i, k}$ de $\mathbf{\Delta} \mathbf{\Delta} \mathbf{B P}$ que não são pagamentos nem recebimentos, ou se cancelam, ou são transportadas para ATVOP I, se forem operacionais, e se agrupam com o LL, e seus respectivos $\Delta_{i}$ são apagados em ATVOP I. Esta ação não altera a soma $\Delta \mathbf{E q C X}$ de suas linhas porque as subvariações de $\Delta_{i}$ continuam a contribuir com o mesmo valor para $\triangle \mathbf{E q C x}$, parte em ATVOP D, parte em ATVOP I, ajustando o Ll como explicamos há pouco. 


\begin{tabular}{|c|c|c|c|c|c|c|}
\hline & & & MATRIZ DFC & & & \\
\hline & Matri & ATVOP I & & Matriz A1 & OP D & \\
\hline & & & & (CAN) & & \\
\hline & & & & (DspS) & & \\
\hline & & & & (DspDpr) & & \\
\hline AJLLOp & & & & (DspF) & & \\
\hline & & & & (DspPCED) & & DRE \\
\hline & & & & (DspDV) & & \\
\hline & & & & Rect & $\operatorname{RecF}$ & 300 \\
\hline & -3.000 & $(L \vee I m b)$ & & LVAmb & & \\
\hline & -1.000 & (PgDspF) & & (PgDspF) & & -1.000 \\
\hline & 1.500 & DspDpr & & $(H R)^{\prime}$ & & \\
\hline & & $L L$ & & & & \\
\hline & & & $\triangle \triangle \mathbf{B P}$ & & & \\
\hline & -10.000 & $-\triangle \mathbf{D R e c}$ & $-[V-\operatorname{Bin} c-\operatorname{Rec} C]$ & $-\gamma+B i h c+\operatorname{Rec} C$ & $\mathrm{RecC}$ & 29.500 \\
\hline & 5.000 & $-\triangle$ DDsc & $-[-D s c D]$ & Dseb & $D s c D$ & 5.000 \\
\hline & 500 & $-\triangle$ PCLD & $-[$ Binc-DspPCLD] & -Binc+DspPCLD & & \\
\hline & -3.000 & $-\triangle \mathbf{E}$ & $-[\mathrm{Co}-\mathrm{CMV}]$ & elo+cys & & \\
\hline & -2.000 & $-\triangle \mathbf{D s p A}$ & $-[N D s p A-A p p D s p A]$ & $-N D s p A+A p p D s p A$ & $-N D s p A$ & -2.600 \\
\hline & 13.000 & $\triangle \mathbf{F}$ & {$[-\mathrm{PgF}+\mathrm{Co}]$} & $-P g F+6 \sigma$ & $-P g F$ & -10.000 \\
\hline & -700 & $\triangle \mathbf{I R}$ & {$[-P g|R+| R]$} & $-P g \mid k+\not R K$ & - PgIR & -2.000 \\
\hline & -7.000 & $\triangle \mathbf{S}$ & {$[-P g S+D s p S]$} & - PgS + Dsps & -PgS & -21.000 \\
\hline$\triangle \mathbf{B P}$ & Matr & z ATVINV & & Matriz A & VINV & \\
\hline & -5.000 & - Smb & $-[A q \mid m b-B i m b]$ & $-A q 1 m b+B i n t b$ & -Aglmb & -20.000 \\
\hline & & & & & Vimb & 15.000 \\
\hline & -1.500 & $-\triangle D p<A c$ & $-[B D p r-D s p D p r]$ & $-B p p r+D s p D p r$ & & \\
\hline & 10000 & DEmpr & {$[-P g D s p F+N E+D s p F]$} & $N E+D S p F$ & $N E$ & 10.000 \\
\hline & Matr & z ATVFIN & & Matriz A & VFIN & \\
\hline & Toser & $C \mathrm{C}$ & & Aunt & Aumc & 10.000 \\
\hline & 2.400 & & & - DDVd & - DDvd & -1.500 \\
\hline & 11.700 & $\triangle \mathbf{E q C x}$ & & & $\triangle \mathbf{E q C x}$ & 11.700 \\
\hline$\triangle \mathbf{E Q C X}$ & 5.600 & Sd Inic & & & Sd Inic EqCx & 5.600 \\
\hline & 17.300 & $\mathrm{Sd} \mathrm{F} \mathrm{EqCx}$ & & & Sd F EqCx & 17.300 \\
\hline
\end{tabular}

